\title{
Legitimation der Folter in der Rechtsprechung zum Asylrecht
}

»Nach der Machtübernahme durch das Militär am I 2.9. 1980 hat sich die Sicherheitslage in der Türkei allgemein ganz erheblich gebessert. Jedermann kann den Schutz der staatlichen Ordnungsorgane anrufen, und diese schreiten auch ein. Wo früher oft das Recht des Stärkeren herrschte, gelten jetzt wieder die verfassungsmäBigen Grundsätze der Gleichheit aller vor dem Gesetz. «' Diese positive Einschätzung der Rückkehr zur kemalistischen Entwicklungsdiktatur ${ }^{2}$ in der Türkei durch den VGH München und die Qualifizierung der Volksabstimmung über die neue türkische Verfassung vom 7. I I. 1982,3 bei der sicherheitshalber Wahlumschläge ausgehändigt wurden, die die Farbe des Wahlzettels durchscheinen ließen und damit Rückschlüsse auf das Votum eines jeden Stimmberechtigten erlaubte, als »ein guter Anfang zur demokratischen Entwicklung “4 legen Zeugnis ab von der Sensibilität bundesrepublikanischer Institutionen gegenüber der politischen Situation in dem NATO-Mitgliedsstaat Türkei. Weder die generelle Untersagung politischer und gewerkschaftlicher Betätigungen' sowie die brutale Unterdrückung der kurdischen Minderheit bis hin zum Verbot, die eigene Muttersprache zu benutzen, ${ }^{6}$ noch die Tatsache, daß mehr als 40000 Inhaftierte von der Militärjunta selbst als politische Häftlinge deklariert werden 7 und allein für 198315800 politische Prozesse vor den Militärgerichten geplant sind, bei denen in ca. 3000 Fällen die Todesstrafe gefordert werden kann, ${ }^{8}$ bewirkte bislang eine grundlegende Änderung der Entscheidungspra-

I VGH München (Informationsbrief Ausländerrecht 1982, I62 L) stellvertretend für die große Zahl der abgelehnten Asylgesuche türkischer Staatsangehöriger.

2 So Klaus Liebe, Zurück zu Kemal Atatürk. Die Türkei nach dem Verfassungsreferendum, Das Parlament, Nr. I v. 8. I. 1983 , S. 1 I. Vgl. in diesem Zusammenhang auch den instruktiven Literaturbericht von Dietrich Thränhardt, Die Türkei. Eine militärische Republik, Teil I, Politische Vierteljahresschrift Literatur 2/1982, S. I $99 \mathrm{ff}$.

3 Vgl. hierzu die vom Bundesvorstand der FIDEF herausgegebene Dokumentation über den Verfassungsentwurf der Militärjunta in der Türkei, Düsseldorf 1982 .

4 So wörtlich Außenminister Genscher in Ankara, Frankfurter Rundschau vom 22. 11, 1982.

5 Vgl. hierzu nur H. E. Schmitt-Lermann, Die politische Strafjustiz des türkischen Militärregimes. Ergebnisse einer Informationsreise nach Istanbul und Umgebung vom 18.-22. 3. 1982, München 1982 .

$6 \mathrm{Zu}$ erwähnen ist in diesem Zusammenhang die besondere asylrechtliche Stellung von Ausländern kurdischer Abstammung aufgrund des Kurdenabkommens von 1928 (Art. I A Nr. I Genfer Flüchtlingskonvention). Danach ist auch heute noch von einer Gruppenverfolgung dieses Bevölkerungsteils Syriens, Iraks und der Türkei auszugehen, die obne den Nachweis individueller Verfolgungsmaßnahmen die Anerkennung von Kurden als politisch Verfolgte i. S. d. Art. 16 II 2 GG bzw. die Einräumung des Flüchtlingsstatus nach der Genfer Konvention nach sich ziehen muß. Ausf. dazu O. Kimminich, Die aktuelle asylrechtliche Bedeutung des Kurdenabkommens von 1928 , AWR-Bulletin $1981,53 \mathrm{ff}$. A. A. VGH Mannheim, DÖV 198 I, $546 \mathrm{~m}$. krit. Anm. Rühmann; VGH Kassel, Informationsbrief Ausländerrecht 1982, $98 \mathrm{~m}$. Anm. Strate. Vgl. auch VG Wiesbaden, Informationsbrief Ausländerrecht 1981, 332.

7 So der türkische Justizminister Cevdet Mentes in einem Interview mit der Zeitung Tercüman, Frankfurter Rundschau vom 17. 8. 1982; vgl. ferner Frankfurter Rundschau vom 10. 9. 1982 unter Bezugnahme auf ein am Vortag in Ankara herausgegebenes Kommunique.

8 Vgl. Frankfurter Rundschau vom I I. I. 1983. 
xis des Bundesamtes für die Anerkennung ausländischer Flüchtlinge. Noch immer bilden die Ablehnungsbescheide, deren Bestandskraft im allgemeinen die Durchsetzung aufenthaltsbeendender Maßnahmen nach sich zieht und den Tod des Abgewiesenen zur Folge haben kann, ${ }^{9}$ den Regelfall. Vor diesem Hintergrund ist es nicht verwunderlich, daß das Bundesamt in Zirndorf die schwere Folterung eines Türken für asylrechtlich irrelevant hielt und als bloße Schutzmaßnahme qualifizierte, "um ihn und seine Gesinnungsgenossen vor weiteren Staatsverleumdungen abzuhalten $4 .{ }^{\circ}$ Zur Abstützung dieser Argumentation konnte das Bundesamt auf Entscheidungen bundesrepublikanischer Verwaltungsgerichte zurückgreifen.

\section{Die Folterrechtsprechung des VGH Kassel und VGH Mannheim}

Die vorgebliche asylrechtliche Bedeutungslosigkeit bereits erlittener oder bei einer Rückkehr in das Heimatland zu erwartender Folterung türkischer Staatsangehöriger ist durch mehrere Entscheidungen des VGH Kassel und VGH Mannheim zum Asylanspruch kurdischer Asylbewerber der breiteren Öffentlichkeit bekannt geworden.

In dem vom VGH Kassel entschiedenen Fall ${ }^{\mathrm{II}}$ hatte sich der Kläger u. a. darauf berufen, er sei im Jahre 1977 dreimal beim Verteilen der kurdischen Zeitschrift »Roja Välat «, die inhaltlich für ein freies und unabhängiges Kurdistan eingetreten ist, verhaftet und anschließend gefoltert worden. Der VGH Kassel vermutet (»... ist es durchaus wahrscheinlich ....) jedoch die maßgeblichen Motive für die Festnahme in der Verfolgung kriminellen Unrechts, das in dem Verbot separatistischer Bestrebungen in Art. I4I, $4_{2}$ türkStGB ${ }^{12}$ seinen Niederschlag gefunden habe, und sieht ihre Vornahme allein »im Zuge strafrechtlicher Ermittlungen«. Weiter heißt es: »Wenn es sodann während der Inhaftierung zu den ... geschilderten Folterungen gekommen ist, geschah dies ebenfalls in dem Bestreben der Polizisten und Jandarmas, ein als strafbar angesehenes Verhalten des Klägers aufzuklären und einer Wiederholung, nämlich einer weiteren Verbreitung der >Roja Välat` vorzubeugen." Auch wenn Folterungen "sich als erhebliche Verletzungen der körperlichen Integrität dar(stellen) und ... gemeinhin als eindrucksvolles Beispiel menschenrechtswidriger Behandlung " gelten sowie als "zweifellos asylrechtlich relevante Beeinträchtigungen und Verletzungen des menschlichen Körpers « eingeordnet werden können, sind sie nach Ansicht des VGH Kassel gleichwohl nicht in jedem Fall als politische Verfolgung anzusehen. Im übrigen habe der Kläger weder glaubhaft gemacht noch sei aus sonstigen Quellen ersichtlich, daß die Mißhandlungen aus einem anderen Grund erfolgt sind als dem, »die Ermittlungen . . . zu fördern « und den Kläger von einem weiteren Einsatz für die Zeitschrift abzuhalten. ${ }^{3}$

Die in der Spruchpraxis des VGH Kassel vorgenommene Einordnung der Folterungen als Mittel der Sachverhaltsaufklärung und polizeilichen Gefahrenvorsorge ist durch eine gleichfalls einen kurdischen Asylbewerber betreffende Entscheidung des

9 Vgl. z. B. den Fall des Türken Sevim Akbas (Frankfurter Rundschau vom 18.8. 1982), dem in der Schweiz Asyl verweigert worden war. Nach erfolgter Abschiebung in die Türkei wurde er dort zu Tode gefoltert.

Io Zitiert nach Jürgen Roth, Kotau vor der Junta - billiger Trost für Verfolgte, Frankfurter Rundschau vom 1. 12. 1982 .

I I VGH Kassel, Informationsbrief Ausländerrecht 1982, $98 \mathrm{~m}$. Anm. Strate.

I 2 Abgedruckt in Informationsbrief Ausländerrecht 1981, $158 \mathrm{f}$. Vgl. jetzt auch Art. 13 der türkischen Verfassung.

I 3 Ebenso VGH Kassel, Informationsbrief Ausländerrecht 1982, 162. 
VGH Mannheim ${ }^{14}$ bestätigt und um eine weitere Variante ergänzt worden. Danach erklärt sich die Mißhandlung von Kurden, die wegen ihrer politischen Aktivität festgenommen wurden, »jedoch nicht allein daraus, daß sie kurdischer Volkszugehörigkeit sind und sich zu einem politischen Programm bekennen«. Vielmehr »handelt es sich bei der Folter in der Türkei um ein allgemeines kriminalpolitisches Phänomen«. Bei ihrer Anwendung sind nach Ansicht des VGH Mannheim keine prinzipiellen Unterschiede, sondern allenfalls "graduelle Abstufungen « zwischen "politischen« und "gewöhnlichen « Straftätern feststellbar. Sie ist "für Türken und Kurden gleichermaßen Alltag, allgegenwärtig, ohne daß sie sichtbare Bestürzung hervorruft" und - so drängt sich förmlich die Schlußfolgerung auf - im Sinne Carl Schmitts durch stillschweigende Hinnahme als Wille des Volkes letztend lich demokratisch legitimiert. Derjenige, der der Begehung von Taten verdächtig ist, »die sich gegen den Bestand des Staates, gegen die Verfassung oder gegen die Gesellschaftsordnung richten « und - »nicht bloß nach türkischem Rechtsverständnis« - dem Bereich der schweren Kriminalität zugerechnet werden, läuft um so eher Gefahr, »in die Nähe potentieller Kapitalverbrecher gerückt und von Sicherheitsorganen, denen hartes Durchgreifen oberste Richtschnur ist«, entsprechend (d. h. auch mit Folter) behandelt zu werden. So fällt es dem VGH Mannheim auch nicht mehr schwer, »das Phänomen, daß Folter und Mißhandlung relativ stark gerade im Vorfeld des politischen Strafrechts verbreitet sind, nicht zuletzt auf kriminaltechnische Besonderheiten « zurückzuführen. Das Ziel, Organisationen, die verdächtig sind, Angriffe auf den Staatsbestand oder die Verfassungs- und Gesellschaftsordnung vorzunehmen, zu zerschlagen, läßt sich danach nur durch Ausschaltung der "führenden Kräfte" erreichen. "Vor diesem Hintergrund dient die Folter, der tatsächliche oder vermeintliche Geheimbündler unterworfen werden, als Mittel, um durch die gewaltsame Erzwingung von Aussagen Erkenntnisse über den organisatorischen Aufbau und die Personalstruktur der Vereinigung zu erlangen. Mit politischer Verfolgung hat dies nichts zu tun.»

\section{II. „Politische Verfolgung* als Grundrechtsvoraussetzung des Art. 16 II 2 GG}

Nach Art. I6 II 2 GG genießen "politisch Verfolgte " Asylrecht in der BRD. Dem Zufluchtsuchenden wird ein Rechtsanspruch auf Schutz vor Ausweisung, Abschiebung und Auslieferung eingeräumt. Bewußt haben die Verfassungsväter und -mütter (immerhin 6!) eine nähere Präzisierung dessen, was als politische Verfolgung zu werten ist, unterlassen. Der im Parlamentarischen Rat vorgetragenen Ansicht des Abg. von Brentano (CDU), der keinen Grund sah, Ausländern, "die aus ihrer Heimat nach Deutschland gekommen sind, weil sie sich in ihrer Heimat aktiv gegen die Demokratie eingesetzt haben«, in Deutschland »unbedingt« Asylrecht zu geben, wurde von dem SPD-Abgeordneten Wagner entgegengehalten, daß der Versuch, das Asylrecht inhaltlich einzuschränken und seine Gewährung "von unserer eigenen Sympathie oder Antipathie" und von der politischen Gesinnung des Flüchtlings abhängig zu machen, bereits als "Beginn des Endes des Asylrechts überhaupt « zu werten sei. ${ }^{\text {'s }}$ Der Abgeordnete Renner (KPD) hat schließlich darauf verwiesen, daß aus politischen Gründen Zufluchtsuchende in jedem Fall von der Sicht des Herkunftslandes - und sei es ein totalitäres Regime wie z. B. seinerzeit Spanien - als

14 VGH Mannheim, Informationsbrief Ausländerrecht 1982, 255 .

15 Die Debatte ist veröffentlicht in: Herbert Spaich (Hg.), Asyl bei den Deutschen, Reinbek 1982, S. $18 \mathrm{ff}$. (29). 
Kämpfer gegen die Demokratie eingeordnet würden. Er wandte sich gegen eine Einengung des Begriffs des politischen Emigranten und erinnerte an die historische Erfahrung mit der Fähigkeit der Nationalsozialisten, diesen Begriff regelmäßig mit dem des kriminellen Flüchtlings zu vermengen. ${ }^{16}$ Der Parlamentarische Rat ist dieser Auffassung mehrheitlich gefolgt und hat daher eine Legaldefinition asylrechtsrelevanter Verfolgung unterlassen. Entsprechend weit ist Art. I6 II 2 GG auszulegen.

An Versuchen, trotz dieser grundsätzlichen Entscheidung des Verfassungsgebers für. einen extensiv zu interpretierenden Begriff des "politisch Verfolgten « den Anwendungsbereich des Art. 16 II 2 GG einzuengen und eine Revision dieses Grundrechts einzuleiten, hat es nicht gefehlt. Anlaß hierzu bot der ab Mitte der 7oer-Jahre festzustellende überdurchschnittlich starke Anstieg der Asylbewerberzahlen, der aber zwischenzeitlich durch immense aufenthalts- und verfahren'srechtliche Restriktionen ${ }^{17}$ abgeblockt wurde. Die rechtspolitische Diskussion kulminierte in der Erklärung des Art. 16 II 2 GG zur "verfassungswidrigen Verfassungsnorm ". ${ }^{18}$

Es ist das Verdienst von E. Paul, ${ }^{19}$ in jüngster Zeit die historischen Beweggründe für die Schaffung des Asylrechts vergegenwärtigt und darauf hingewiesen zu haben, daß dem Grundrecht aus Art. I6 II 2 GG keineswegs ein Leitbild zugrunde liegt, das sich an persönlichen Schicksalen - etwa von Marx in London, Lenin in der Schweiz oder Willy Brandt in Norwegen und Schweden - orientiert. ${ }^{20}$ Die entscheidende Grundlage für die Schaffung des Asylrechts bildet die historische Erfahrung der großen Zahl namenloser Flüchtlinge, die den Verfolgungen des NS-Regimes entrinnen wollten und an den Grenzen der europäischen Nachbarstaaten abgewiesen oder wieder an die deutschen Dienststellen ausgeliefert wurden. ${ }^{21}$

Zur Bestimmung dessen, was "politische Verfolgung * konkret bedeutet, kann nicht auf eine statische Begriffsdefinition zurückgegriffen werden. ${ }^{22}$ Vielmehr ist Art. I 6 II ${ }_{2}$ GG als interpretationsbedürftige »offene Norm «, die freilich durch die jahrzehntelange Entscheidungspraxis von Bundesamt und Rechtsprechung eine hinreichende und umfassende Konturierung erfahren hat, durchaus auch der Beeinflussung durch politische Entwicklungen und Geschehnisse ausgesetzt, freilich ohne durch diese Einbindung in den dynamischen politischen Prozeß zu einem nach Opportunitätserwägungen einsetzbaren Instrumentarium degradiert werden zu dürfen. Im Gegenteil: Art. 16 II 2 GG als Ausdruck und Ergebnis spezifischer historisch-politischer Erfahrungen verbietet es, den verfassungsrechtlichen Asylanspruch von der Herkunft und politischen Gesinnung des Verfolgten oder vom politischen System, das im Verfolgerstaat herrscht, abhängig zu machen. Auch eine Beschränkung auf bestimmte "asylwürdige " Rechtsgüter ist nicht gerechtfertigt. Den Schutz des Art. 16 II 2 GG genießt demnach jeder, »der aus politischen Gründen Verfolgungsmaßnahmen mit Gefahr für Leib und Leben oder Beschränkungen seiner persönlichen Freiheit ausgesetzt wäre . . oder - allgemein gesagt - politische Repressalien zu erwarten hätte... Voraussetzungen und Umfang des politischen Asyls sind wesentlich

16 Renner, in: Spaich (0. Anm. 15), S. 35.

17 Vgl. hierzu B. Huber, Die Entwicklung des Ausländer- und Arbeitserlaubnisrechts im Jahre 1981, NJW 1982, 1914 mit weiteren Rückverweisungen.

I 8 So ausdrücklich W. Philipp, NJW 1981, 1857; dagegen M. Sachs, NJW 1981, 2608 und D. Hömig, Verwaltungsrundschau $1982,147$.

19 Asylrecht im Zwielicht?, Zeitschrift für Ausländerrecht 1982, 184.

20 So aber der Vizepräsident des BVerfG Zeidler in: Praktizierte Humanitas: Weltproblem Flüchtlinge eine europäische Herausforderung, Baden-Baden 1981, S. 2 I.

21 Ausführlich dazu E. Paul (o. Anm. 19), S. $184 \mathrm{ff}$.

22 Mit Recht ablehnend gegenüber der Forderung nach einer Legaldefinition u. a. O. Kimminich, Der Aufenthalt von Ausländern in der Bundesrepublik Deutschland, Baden-Baden 1980, S. 14I f. m. w. N. 
bestimmt von der Unverletzlichkeit der Menschenwürde, die als oberstes Verfassungsprinzip nach der geschichtlichen Entwicklung des Asylrechts die Verankerung eines weitreichenden Asylanspruchs im Grundgesetz entscheidend beeinflußt hat. ${ }^{23}$ Dies ist der historisch gewachsene und zugleich als Wesensgehalt (Art. 19 II GG) geschützte unantastbare Kernbereich des Art. 16 II 2 GG. Die "Offenheit dieses Grundrechts und damit letztendlich die Beeinflussung seines Inhalts durch politische Prozesse besteht demnach allein darin, gegebenenfalls seine Anwendung auf bisher nicht gekannte neue Tatbestände politischer Verfolgung zu erstrecken. ${ }^{24}$ Das subjektiv öffentliche Recht auf Asylgewährung setzt nach der Rechtsprechung des BVerfG tatbestandlich voraus, daß die menschenrechtswidrige Behandlung "aus politischen Gründen" erfolgt. VGH Kassel und VGH Mannheim sehen die von den türkischen Stellen gegenüber den kurdischen Asylbewerbern wegen deren Bereitschaft, gegebenenfalls auch mit Gewalt durchzusetzende separatistische Bestrebungen zu unterstützen, vorgenommenen bzw. zu erwartenden Verfolgungsmaßnahmen als rein strafrechtlich motiviert an. Folglich wird auch den im Verlauf der Ermittlungen praktizierten oder bei Rückkehr zu befürchtenden Folterungen jegliche asylrechtliche Relevanz aberkannt. Als entscheidender Maßstab wird letztendlich der »kriminelle Unrechtsgehalt« der angelasteten Delikte zugrundegelegt und damit dem in ihnen zum Ausdruck kommenden Angriff auf den Bestand des türkischen Staates die politische Qualität der Strafverfolgungsmaßnahmen abgesprochen. Mit dieser Sichtweise wird jedoch die Tat des Verfolgten zum entscheidenden Kriterium für die Zuerkennung des Asylrechts, nicht aber die allein maßgebliche Zielrichtung des Verfolgerstaates. Die Handlungen des Asylbewerbers im Heimatland, die möglicherweise mit der freiheitlich-demokratischen Grundordnung des Grundgesetzes nicht in Einklang stehen, haben auf die Gewährung des Asylrechts keinerlei Einfluß. ${ }^{25}$ Die Vorschriften des politischen Strafrechts - hierzu gehören die Normen, wegen derer die Kläger in den Verfahren des VGH Kassel und VGH Mannheim festgenommen wurden und nach der Rückkehr eine Verhaftung zu befürchten haben - dienen allein politischen Zielen. ${ }^{26} \mathrm{Ihr} Z$ weck besteht gerade in der Unterdrückung bestimmter politischer Meinungen und Handlungen und der Einflußnahme auf den politischen Prozeß. Zumindest wenn im Zusammenhang mit der strafrechtlichen Verfolgung menschenrechtswidrige, den rechtsstaatlichen Grundsätzen des Grundgesetzes widersprechende $\mathrm{Maßnahmen}$ ergriffen werden wie z. B. Folterungen oder sonstige Beeinträchtigungen der physischen und psychischen Integrität und kein rechtsstaatliches System zum effektiven Schutz vor solchen Maßnahmen existiert, ${ }^{27}$ sind diese Mißhandlungen als asylrechtsrelevante Verfolgung im Sinne des Art. I6 II 2 GG zu werten. ${ }^{28}$

Die Tatsache, daß in der Türkei die systematische Anwendung von Foltermethoden

$23 \mathrm{BVerfGE}_{54,341}=$ NJW 1980, 264r. Rechtsstaats- und Menschenrechtswidrigkeit der Verfolgung wird u. a. auch von I. v. Pollern (Das moderne Asylrecht, Berlin 1980, S. 284) als Auslegungsmaßstab für Art. 16 II 2 GG herangezogen. Ebenso D. Franke, Politisches Delikt und Asylrecht, Königstein I979, S. 6r. Kritisch hierzu Ch. Gusy, Asylrecht und Asylverfahren in der Bundesrepublik Deutschland, Königstein $1980, \mathrm{~S} .92 \mathrm{ff}$.

${ }_{24}$ Vgl. dazu nur O. Kimminich, Verhandlungen des 53. Deutschen Juristentages Berlin 1980, München $1980, \mathrm{~B} 37$.

25 So auch D. Franke (o. Anm. 23), S. 67 ff.

26 Auch das „gemeine« Strafrecht dient - soweit es private Rechtsgüter schützt - im Endeffekt gleichfalls politischen Zwecken. Ansatzpunkt für staatliches Handeln ist jedoch insoweit nicht eine politische Ansicht per se. Vgl. Ch. Gusy (o. Anm. 23), S. 12 I.

27 Hierzu gehören auf keinen Fall die auf Foltervorwürfe gestützten »Schaufenster-Anklagen $\propto$ gegen Mitglieder türkischer Sicherheitsorgane, da deren Zielrichtung allein außenpolitischer Natur ist.

28 So auch im Gegensatz zu VGH Kassel und VGH Mannheim ausdrücklich OVG Saarlouis, Informationsbrief Ausländerrecht und VG Hamburg, Informationsbrief Ausländerrecht 1982, 101 und $163 \mathrm{~L}$. 
tagtäglich erfolgt und hierbei kein Unterschied zwischen Personen, denen politische Delikte angelastet werden, und Beschuldigten, die »gemeiner « Straftaten verdächtig sind, getroffen wird, ${ }^{29}$ rechtfertigt keinesfalls die Verneinung des Asylanspruchs. Vielmehr läßt dies den Schluß zu, daß mit dieser Demonstration staatlicher Macht eine Stabilisierung und Festigung des bestehenden politischen Systems erfolgen soll. Sie beinhaltet damit bereits politische Verfolgung im Sinne des Art. I6 II 2 GG.

Vor den Richtern, die die besprochenen Entscheidungen gefällt haben, hätten weder die Mitglieder des 20. Juli 1944 oder der "Weißen Rose« noch die Widerstandskämpfer der Arbeiterbewegung oder die große Zahl der namenlosen Flüchtlinge, die den Verfolgern des nationalsozialistischen Regimes durch Flucht entkommen wollten, eine Chance gehabt, jemals Asyl zu erlangen. Die Entscheidungen des VGH Kassel und VGH Mannheim entkleiden Art. 16 II 2 GG seiner Funktion, gegenüber Menschenrechtsverletzungen totalitärer Systeme effektiven Verfolgungsschutz zu gewähren. Es bleibt zu hoffen, daß das BVerwG dieser zumindest partiellen Abschaffung des Asylrechts einen Riegel vorschiebt und damit der Spruchpraxis anderer bundesrepublikanischer Verwaltungsgerichte zur Folter als asylbegründender Maßnahme folgt ${ }^{29 a}$. Andernfalls gewinnt dieses Grundrecht tendenziell eine Bedeutung, wie sie Art. 16 S. 3 der türkischen Verfassung ("Niemand darf gefoltert oder mißhandelt werden «) in der türkischen Realität zukommt.

\section{Die Spuren der Vergangenheit}

Die Bedeutung der Aussagen des VGH Kassel und VGH Mannheim zur asylrechtlichen Relevanz von Folterungen bleibt nicht auf den Bereich des Ausländerrechts beschränkt. Indem sich die erkennenden Richter in breiter Ausführlichkeit über die geschilderten Mißhandlungen auslassen und hierbei zugleich eine qualitative Bewertung dieser Maßnahmen vornehmen, sind Denk- und Bewußtseinsstrukturen transparent geworden, die mit den rechtsstaatlichen Grundsätzen der bundesrepublikanischen Verfassung nicht in Einklang zu bringen sind. Die geschichtlichen Erfahrungen mit dem nationalsozialistischen Gewaltregime, die zur Schaffung des Grundgesetzes mit seinem Grundrechtskatalog und seinen rechtsstaatlichen Sicherungen geführt haben, scheinen fünfzig Jahre nach der Machtergreifung Hitlers bei einem Teil der Justiz in Vergessenheit geraten zu sein. Vor der Argumentation dieser Gerichte versagt die gebotene analytische Distanz: Es ist einfach erschütternd, wie Folter als "geeignetes Mittel der Sachaufklärung ${ }^{3{ }^{3 \circ}}$ erklärt und zugunsten übergeordneter "staatlicher Zwecke« als kriminaltechnisch geboten eingestuft wird.

In Anlehnung an Ilse Staff muß für die aufgezeigte Rechtsprechung zur Folter die Feststellung getroffen werden, daß die Würde des Menschen auch durch Richtersprüche verletzt werden kann, "wenn nicht das Individuum, sondern die Staatsraison zum Maßstab staatlichen Handelns wird « ${ }^{31}$.

Bertold Huber

29 Vgl. aber die Darstellung des OVG Saarlouis (Informationsbrief Ausländerrecht 1982, 306), wonach bei Verfolgung "politischer « Delikte die Mißhandlungen eine besondere Intensität erreichen.

29a Einen entscheidenden Schritt in diese Richtung bilden die neuesten, nach Manuskriptabschluß getroffenen Entscheidungen des BVerfG (1 BvR 109/82; I BvR 990/82) zum Auslieferungsverkehr mit der Türkei. Vgl. Frankfurter Rundschau v. 31.3.1983. Lt Pressemeldungen (Südd. Zeitg. v. 18. 5. 83) entschied das BVerwG, daß Folter zwar generell einen "eklatanten Verstoß gegen die Menschenwürde" darstelle, asylrechtlicher Schutz aber nur bei (im Einzelfall zu prüfender) politisch motivierter Verfolgung gewählt werden dürfe (Az.: I BVR 866/82).

30 G. Strate, Informationsbrief Ausländerrecht 1982, 100 (101).

3 I. Staff, Justiz im Dritten Reich, Frankfurt am Main 1978, S. 223. 